\title{
The Industrial Pharmacy Lab: New Concepts in Powder and Process Technology
}

\author{
Gabriele Betz*
}

\begin{abstract}
The Industrial Pharmacy Lab (IPL) was founded in 2001 and has been awarded the NETS Award and NETS Special Award of the Gebert Rüf Foundation, Basel in 2004. The IPL is a turning platform between the university and the pharmaceutical industry at the Pharma Hub Basel with the focus on research in powder technology, dosage form design and scale-up. The IPL works with industrial-sized equipment and applies new and state-of-the-art technologies to the development of robust formulations. In the present article the research fields granulation process control, formulation of low water soluble drugs, modified release, and impact of the filling process on protein solutions are introduced and critical parameters, sources of variability, and scale-up problems are identified in order to suggest and provide a rational and optimal formulation and process for pharmaceutical dosage form design.
\end{abstract}

Keywords: Granulation process control · Low water-soluble drugs · Modified release · PAT initiative ·

Protein aggregation

\section{Introduction}

The Industrial Pharmacy Lab (IPL) of the Institute of Pharmaceutical Technology, University of Basel was founded in 2001 as a turning platform between the university and the pharmaceutical industry at the Pharma Hub Basel. In close cooperation with the pharmaceutical industry, this is a very unique concept and was awarded the NETS Award (New Entrepreneurs in Technology and Science) and the NETS Special Award of the Gebert Rüf Foundation, Basel in 2004.
The mission of the IPL is to focus on research in powder technology, dosage form design and scale-up. This research is very close to today's needs of the pharmaceutical industry; looking for robust formulations and process technologies, which should shorten the development time and increase the product quality. A close cooperation with the pharmaceutical industry is a prerequisite to be able to do studies in the area of scale-up aiming to understand and control pharmaceutical processes, which is in agreement with the basic tenet of 'quality by design' of the FDA's PAT (Process Analytical Technology) initiative.

Over $80 \%$ of the active drugs that are formulated to produce systemic effects in patients are marketed as solid dosage forms, due to the great acceptance of patients to take tablets. Furthermore, more than $40 \%$ of newly discovered drugs are poorly water soluble, and there is no universal formulation technique for any sparingly water-soluble drug. Therefore, the pharmaceutical industry is investing vast amounts of money in research and development of this specific area. However, the severe problem of the pharmaceutical industry is to increase productivity and creativity at the same time, and therefore they seek collaborations with universities. Based on this mutual interest between university and pharmaceutical industry the Industrial Pharmacy Lab was founded as a turning platform between the two sides.
The IPL applies new and state-of-theart technologies and equipment to the development of robust formulations leading to reliable manufacturing processes for high volume production. The IPL works with industrial-sized equipment such as a compaction simulator (sponsored by Pfizer, Freiburg), different types of tableting machines (sponsored by Korsch, Berlin), equipment for manufacturing granules and pellets (sponsored by Glatt, Binzen), a capsule filling machine (sponsored by Pfizer, Freiburg), a roller compactor (sponsored by Fitzpatrick, Sint-Niklaas, Belgium) and a sterile ampoule filling line (sponsored by Novartis, Basel).

\section{Research Fields}

Tablets are complex systems and the behaviour of disordered particulates under pressure is still far from being well understood, especially with high-speed compression cycles similar to presses used in industry. Manufacturing problems of tablets are usually discovered towards the end of the development process when compression runs are studied for the first time. The use of a compaction simulator in the early stage of development is a significant benefit for the product development process.

In the pharmaceutical process of tablet production, moist agglomeration is commonly applied to powder mixtures in order
F-Mail: Gabriele.Betz@unibas.ch

www.pharmtech.unibas.ch 
to improve powder characteristics such as flow and compressibility. The desired properties of the granules are controlled by a combination of formulation design (choosing the starting material and liquid according to its properties) and process design (choosing the type of granulator and the operating parameters). Changing the formulation and/or the process itself is followed by a high number of experiments based on trial and error. Furthermore, a change in the quality of granules can have an influence on the compression runs, especially with high speed cycles and therefore on the properties of the final tablet.

The aim of the research group working in the Industrial Pharmacy Lab is to understand and, in a second step, to control pharmaceutical processes in the field of dosage form design in order to develop robust formulations and to increase the product quality. It is essential that the 'art of formulation' is converted into science, taking into account percolation theory and the laws of physical pharmacy. This is in agreement with the science-driven PAT initiative, which is an effort to facilitate the introduction of new technologies into the pharmaceutical industry. The vision is to investigate, design, and develop processes that can consistently ensure a predefined quality at the end of the manufacturing process.

In the present research overview of the IPL, tools for robust dosage form design are introduced in the following areas:

- Granulation Process Control (2.1.)

- Formulation of Low Water-soluble Drugs (2.2.)

- Modified Release (2.3.)

- Impact of the Filling Process on Protein Solutions (2.4.)

In all the four areas, it is important to identify critical parameters, non-robust formulations, sources of variability and scaleup problems in order to suggest and provide a rational and optimal formulation and process for pharmaceutical dosage form design.

\subsection{Granulation Process Control}

The research work in the area of granulation process control gave valuable insight into process and formulation design. Power consumption, temperature recording, and tensile strength measurements during the granulation process led to an in-process control device which records and analyses the power consumption profile taking into account the properties of the starting material, such as particle size, surface, solubility, and wettability. Applying the theory of percolation, three percolation thresholds were obtained during the granulation process with mannitol due to a superposition of bond and site percolation effects. In robust formulation design it is important to determine the percolation thresholds for each formulation in order to avoid variations in granule quality.

The compression behaviour of pharmaceutical substances and the final property of the tablet can be significantly influenced by the properties of the starting material and the granulate. In order to maintain the granulate quality, an in-process control device was applied to various tablet formulations. The turning point of the power consumption profile was introduced as the parameter for the cohesiveness of the starting material and used for optimal end-point detection at an early stage. The influence of changing the granulating liquid on the power consumption profile could be predicted by calculating the ratios of the surface tension of the granulating liquids. Furthermore, tensile strength measurements proved that the developed device, measuring the power consumption of the granulator, is an alternative to determining the cohesion of powder particles [1-4].

Temperature recording was also introduced as an efficient in-process control. Furthermore, the ratio of power consumption and temperature recording (TPR) was introduced as a signature of formulation design. The influence of changing the formulation can be predicted by considering particle size, particle surface, water absorption capacity and solubility of the starting material. The TPR-factor was determined to be dependent on formulation design, and not on process design, such as the filling level of the mixer. The filling volume-specific power consumption was also discovered to be independent of the relative filling level. This is an important result for granulation process scale-up and allows the estimation of the power consumption of larger scales before the process is started. The results of the presented work [5][6] have given valuable information about the granulation process and are currently used to develop an artificial neural network for in-process granulation control.

The first results were obtained by applying Kohonen networks [7] and selforganized maps to determine the critical granulation process steps; and furthermore, to avoid them. Application of different techniques for power consumption signal analysis is currently being compared and optimized for the in-process device in cooperation with the Mendeleyev University, Moscow.

\subsection{Formulation of Low Water-solu- ble Drugs}

Based on the excellent results of the research group of Prof. Dr. Hans Leuenberger in powder technology, the research topic is extended to compaction simulation. A compaction simulator - Presster ${ }^{\mathrm{TM}}$ (MCC) - was sponsored by Pfizer (Freiburg, Germany) and allows multi station rotary tablet presses to be simulated using only a minimal amount of tableting material, and to avoid problems towards the end of the development process. Two examples of low water-soluble drugs, mefenamic acid and carbamazepine, belonging to Class II of the bioclassification system, were selected for investigations. In fact, more than $40 \%$ of the discovered drugs fall into these categories and additionally (i.e. in the UK) $41 \%$ count as failures in new drug development due to biopharmaceutical properties, solubility being one of them.

\subsubsection{Mefenamic Acid (MA)}

In order to increase the specific surface of a low water-soluble drug, it is important that the tablet shows an optimal and fast disintegration.

Investigations were performed to optimize the loading volume of mefenamic acid for granules and tablet formulations. The investigation ranged from the placebo formulation up to the loading volume of $75 \%$ (v/v) MA. Characteristics of granules and tablets produced by the fluidized bed granulator and compaction simulator, respectively, were evaluated in order to suggest formulation rules for the use of mefenamic acid in solid dosage forms.

The fastest disintegration time was obtained with 50\% mefenamic acid (Fig. 1) even though mefenamic acid shows low water solubility and wettability. With lower and higher MA content the disintegration time increased.

It can be assumed that the disintegrant particles, such as maize starch, are in close contact with the network of pores which extends throughout the whole tablet. At a concentration lower than the critical amount of disintegrant, the individual particles do not cover all pore sites of the porous network. As the drug substance is hydrophobic, the hydrophilic particles facilitate the water uptake due to hydrophilized pores. For a concentration higher than the percolation threshold, the water uptake is reduced due to the swelling properties of the disintegrant [8-11].

\subsubsection{Carbamazepine}

Carbamazepine (CBZ) exhibits at least four polymorphic forms and a dihydrate. CBZ polymorphs have different crystal structures and exhibit different melting points, chemical reactivity, solubility, and compactibility, all of which can contribute to differences in bioavailability. The pure active substance can contain a mixture of polymorphs and crystal modifications as well as amorphous material. This variation can lead to an alteration in the dissolution profile during storage due to the transformation from one polymorph to the other. Therefore, there is a need to define adequate parameters and properties which must be monitored and controlled in order 


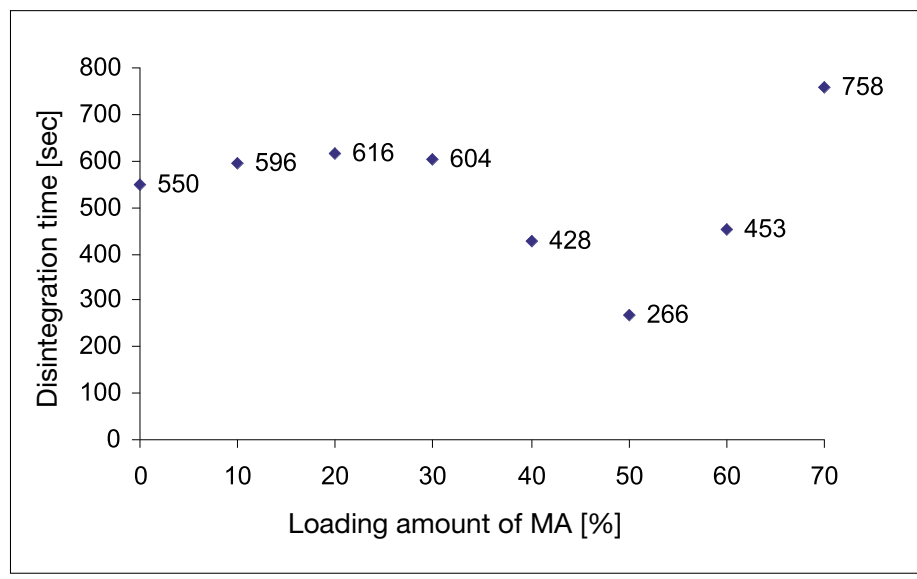

Fig. 1. Disintegration of tablets with increasing loading amount of MA

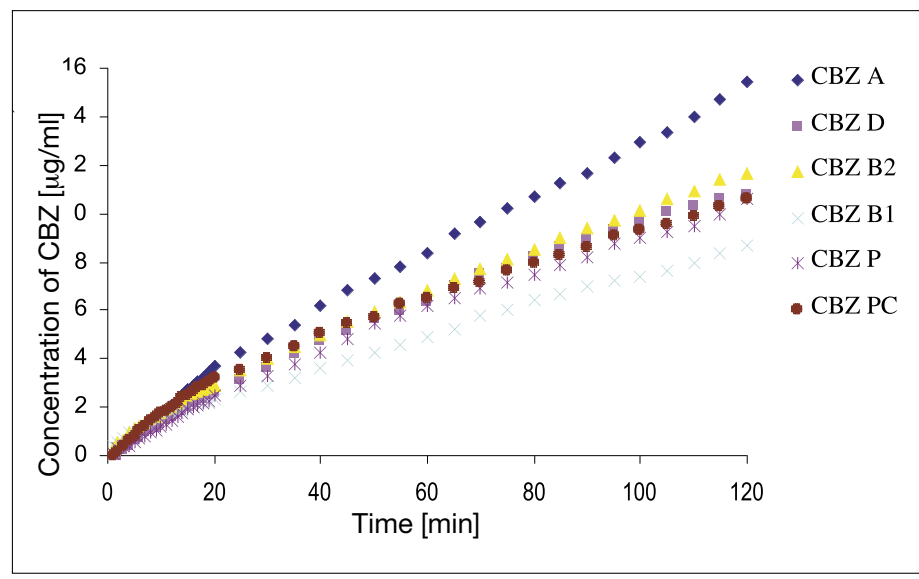

Fig. 2. Dissolution profile during the intrinsic dissolution to maintain the shelf-life stability of CBZ solid dosage forms. The aim of the study was to investigate the influence of variability of commercially available CBZ from different manufacturers on the intrinsic dissolution behaviour and solubility in order to recommend a strategy to maintain product quality by monitoring critical parameters of the bulk drug.

The results obtained with differential scanning calorimetry (DSC) confirmed that all CBZ samples belonged to polymorphic form III (Table 1). The melting point of form I of all commercial samples was determined as ca. $192^{\circ} \mathrm{C}$, and was in agreement with the result obtained for CBZ USP standard.

The solubility of CBZ samples at equilibrium and the intrinsic dissolution data are shown in Table 2.

Anhydrous CBZ (CBZ P, Pc, A, B1, B2) was assumed to undergo a phase transition into the dihydrate form (CBZ D) indicated by the change of slope in the dissolution profile. Based on this result, the intrinsic dissolution rates were calculated from the initial slope of each form after $20 \mathrm{~min}$ (Fig. 2). Although all commercially analyzed samples of CBZ showed the same polymor- phic form, solubility and intrinsic dissolution rates were found to vary among them and to be significantly lower compared to literature data. It is suggested that the intrinsic dissolution kinetics were changing during experiments due to the transition of the anhydrous form of CBZ [12] to the dihydrate.

All these variations can be attributed to various manufacturing processes and are most likely to result from different drying and/or grinding regimes in the final stage of the manufacture.

The dihydrate dissolution profile showed a change of slope as well, which is not in agreement with literature data. A possible reason is the impurity of the dehydrate itself, and thus further investigations are needed.

\subsection{Modified Release}

Modified release dosage forms optimize the therapeutic efficiency of drug treatment by minimizing drug fluctuations in the systemic circulation by extending the duration of action. Thus dose frequency can be reduced, resulting in an improvement of dose discipline for the patients.
Modified release from matrix systems prepared from insoluble polymer or lipid matrices is determined by the pore structure of the system. Drug particles dispersed in the insoluble matrix become available as the solvent enters the matrix and dissolves the particles. In the work of Bonny [13-15] fractal dimensions were used to describe irregular surfaces and pore structures of matrix systems consisting of ethylcellulose or hydrogenated castor oil and caffeine. The pore structure remaining after different amounts of drug were released was described by the volume and surface fractal dimension. The volume fractal dimension $\mathrm{D}_{\mathrm{V}}$ turned out to be a useful parameter to characterize the pore structure generated by the dissolution of the drug. The latter was influenced by the grain size of the incorporated drug and decreased in an approximately linear way with increasing leaching time of the corresponding matrix. The preparation of such matrix systems is very simple and inexpensive, and can be performed by direct compression of the mixture or by compression after granulation. Applying the concepts of percolation theory [16] to the diffusion of a drug out of a matrix, it was demonstrated
Solid-solid transformation $\mathrm{III} \rightarrow \mathrm{I}\left[{ }^{\circ} \mathrm{C}\right]$

$\begin{array}{ll}\text { CBZ USP } & 176.57 \\ \text { CBZ Da } & 60.54-89.05 \\ \text { CBZ P } & 184.96 \\ \text { CBZ A } & 174.10 \\ \text { CBZ B } & 176.52 \\ \text { CBZ B }_{2} & 177.16\end{array}$

\begin{tabular}{ll}
$\begin{array}{l}\text { Melting point of } \\
\left.\text { form I [ }{ }^{\circ} \mathbf{C}\right]\end{array}$ & Enthalpy [J/g] \\
\hline 191.88 & 107.32 \\
191.92 & 92.87 \\
192.38 & 106.31 \\
191.79 & 107.13 \\
192.96 & 108.89 \\
192.91 & 106.98
\end{tabular}

${ }^{a}$ dehydratation

\begin{tabular}{|lll|}
\hline & $\begin{array}{l}\text { IDR } \\
{\left[\mu \mathrm{g} / \mathrm{cm}^{2} / \mathrm{min}\right]}\end{array}$ & $\begin{array}{l}\text { SOLUBILITY } \\
{[\mu \mathrm{g} / \mathrm{ml}]}\end{array}$ \\
\hline CBZ D & 23.6 & 253.6 \\
\hline CBZ P & 18.0 & 207.3 \\
\hline CBZ PC & 20.4 & 237.5 \\
\hline CBZ A & 53.9 & 430.4 \\
\hline CBZ B & 18.5 & 275.1 \\
\hline CBZ B & 23.9 & 254.2 \\
\hline
\end{tabular}


that in a binary system consisting of the drug and the matrix-forming excipient, two percolation thresholds can be determined. The lower percolation threshold $\mathrm{p}_{\mathrm{c} 1}$ corresponds to a critical porosity $\varepsilon_{\mathrm{c}}$ of the matrix, and was determined to be 0.32 for ethylcelluose matrices containing caffeine as drug particles. The upper percolation threshold $\left(p_{c 2}\right)$, however, represents the critical volume fraction of matrix substance which is required to ensure the integrity of the matrix during drug release, and was found to be smaller than 0.29 [15].

Polysaccharides are widely used in pharmaceutical formulation, and in several cases play a fundamental role in modified release control. A novel network-forming starch was used to prepare matrix systems with caffeine, and investigations were performed on its future use in controlled release systems.

Matrix systems were prepared by extrusion with novel starch film containing caffeine in the concentration range of $5-50 \%$. Matrix tablets were prepared by mixing ground starch film with caffeine in respective amounts ( 5 to $50 \%$ ) and pressed into tablets. It was shown that the caffeine release profile followed Higuchi's square root of time law for all the starch disks and tablets, and was in the range of ethylcellulose matrix systems.

The caffeine release profile was largely independent of drug loading, thickness of the delivery system and presence of amylase in the dissolution media, however it depended on the network density of prepared starch disks. Since the latter are made by simply adding different amounts of drug, starch and short-chain amylose, one can adjust the release rate for a given drug loading [17].

\subsection{Impact of the Filling Process on Protein Solutions}

During production, proteins are exposed to various stresses which can cause protein denaturation and inactivation. The objective of the present study was to investigate the effect of shear forces which can occur during filling operations of pharmaceutical solutions with dosing equipment. Such shear forces could have a negative influence on shear sensitive substances and may lower the quality of the final product. In the first part of this work the influence of various parameters during the filling operation such as filling speed, dosing volume and size of pumps was examined using a model protein solution. Furthermore, the influence of rotary piston pumps and peristaltic pumps was compared. In the second part, excipients were tested on their ability to protect the model protein against shear-induced damage. Photon correlation spectroscopy (PCS) and size exclusion chromatography were used to determine the $\mathrm{z}$-average mean and the size distribution of the model protein before and after shearing, as a method to evaluate the degree of denaturation and aggregation. It was found that protein aggregation was induced by rotary piston pumps and not by peristaltic pumps. The smaller the size of the rotary piston pumps the more protein aggregation occurred. The filling speed had a minor influence, and no protective effect could be seen by adding excipients [18].

\section{Future Perspectives}

New projects are currently running on the topic of 'Controlled release tablets containing theophylline prepared by using an instrumented roller compactor' and 'Stabilisation of the glucose blood level in diabetics using network-forming starch formulations'.

Received: December 22, 2005

[1] G. Betz, P. Junker Bürgin, H. Leuenberger, Pharm. Develop. Tech. 2003, 8, 289.

[2] G. Betz, P. Junker Bürgin, H. Leuenberger, Int. J. Pharm. 2003, 252, 11 .

[3] H. Leuenberger, G. Betz, D. Jones, in 'Pharmaceutical Process Scale-up' Ed. M. Levin, Marcel Dekker, Inc., in press.

[4] G. Betz, P. Junker Bürgin, H. Leuenberger, 4th World Meeting on Pharmaceutics Biopharmaceutics Pharmaceutical Technology, Florence, Italy, April 2002.

[5] G. Betz, P. Junker Bürgin, H. Leuenberger, Int. J. Pharm. 2004, 272, 137.

[6] G. Betz, A. Meyer, M. Puchkov, H. Leuenberger, 5th Central European Symposium on Pharmaceutical Technology and Biotechnology, Ljubljana, Slovenia, September 2003.

[7] B. Meyer, M. Puchkov, O. Eichhorst, G. Betz, H. Leuenberger, Pharmaceutical Sciences Fair, Nice, France, June 2005.

[8] G. Kimura, G. Betz, H. Leuenberger, Pharm. Develop. Tech. 2005, in press.

[9] G. Kimura, M. Puchkov, G. Betz, H. Leuenberger, Pharmaceutical Sciences Fair, Nice, France, June 2005.

[10] G. Kimura, G. Betz, H. Leuenberger. 3rd World Conference on Drug Absorption, Transport and Delivery, Barcelona, Spain, April 2005.

[11] G. Kimura, G. Betz , H. Leuenberger. 12th International Pharmaceutical Technology Symposium, Istanbul, Turkey, September 2004.

[12] S. Sehic, H. Trobradovic, G. Betz, S. Hadzidedic, S. Kocova El-Arini, H. Leuenberger, World Congress of Pharmacy and Pharmaceutical Sciences, Cairo, Egypt, September, 2005.

[13] J.D. Bonny, Ph.D.-thesis, University of Basel, Switzerland, 1992.

[14] J.D. Bonny, H. Leuenberger, Eur. J. Pharm Biopharm. 1993a, 39, 31.
[15] J.D. Bonny, H. Leuenberger, Pharm. Acta Helv. 1993b, 68, 25.

[16] D. Stauffer, A. Aharony, Taylor \& Francis Ltd, 1994.

[17] M. Honegger, R. Müller, F. Innerebner, G. Betz. Pharmaceutical Sciences Fair, Nice, France, June 2005.

[18] G. Betz, U.J. Bausch, H. Leuenberger, Pharmaceutical Sciences Fair, Nice, France, June 2005. 